\title{
El comportamiento de la prensa oficial con respecto al Gobierno Suárez durante las elecciones legislativas de junio de $1977^{1}$
}

\author{
Jezabel MARTÍNEZ FÁBREGAS \\ jmartinez10@us.es \\ Universidad de Sevilla
}

Recibido: 24 de julio de 2014

Aceptado: 19 de enero de 2015

\section{Resumen}

La prensa oficial española tuvo un comportamiento heterogéneo con respecto al proceder del Gobierno Suárez durante las elecciones legislativas de 1977. Ésta es la hipótesis de la que parte un trabajo que, a través del análisis de contenido, estudia el proceder de los textos de opinión publicados en los diarios de la prensa oficial entre el 8 y el 18 de junio de 1977, y que demuestra que la prensa oficial abandona en este momento histórico su función propagandística, acogiendo distintos posicionamientos con respecto a la gestión gubernamental, algunos de ellos críticos, asumiendo así su rol de agente político.

Palabras clave: Prensa oficial, transición española, Gobierno Suárez, propaganda, historia del periodismo español.

\section{The Official Press Behaviour with Respect to Suarez Government during the Legislative Elections in June 1977}

\begin{abstract}
The Spanish official press had an heterogeneous behaviour with respect to Suárez Government during the legislative elections in 1977. This is the hypothesis from which this study parts and which, through content analysis, studies the conduct of opinion texts published in the official newspapers between 8th and 18th June 1977, and which shows off that the official press abandoned its propaganda function at this time taking different positions with respect to the governmental management, some of them critical, and assuming, in this sense, its role as a political agent.
\end{abstract}

Keywords: Official press, Spanish transition, Suárez Government, propaganda, History of Spanish Journalism.

\section{Referencia normalizada}

MARTÍNEZ FÁBREGAS, Jezabel (2015): "El comportamiento de la prensa oficial con respecto al Gobierno Suárez durante las elecciones legislativas de junio de 1977". Estudios sobre el Mensaje Periodístico. Vol. 21, Núm. 1 (enero-junio), págs.: 115-130. Madrid, Servicio de Publicaciones de la Universidad Complutense.

Sumario: 1. Introducción. 2. Metodología. 3. Resultados y discusión. 4. Conclusiones. 5. Anexo. 6. Referencias bibliográficas.

\section{Introducción}

La prensa oficial fue diseñada por el régimen franquista para servirle como órgano de propaganda a través del cual inculcar a la población el terror nacional sobre el que se

1 Este trabajo se inserta en el Proyecto de Investigación "El papel de la prensa diaria en la transición democrática. Cobertura informativa y comportamiento político de diarios y periodistas", referencia CSO2012-36774, del Plan Nacional I+D 2013-2015 CSO 2012 36774, dirigido por el catedrático Jaume Guillamet Lloveras (Universitat Pompeu Fabra). 
sustentaba la dictadura franquista. En este sentido, los periódicos oficiales bebían de las consignas enviadas desde la Delegación Nacional de Propaganda (DNP) y, posteriormente, desde el Ministerio de Información y Turismo. Pero, independientemente de la institución que la controlara, ésta debía mantener siempre un discurso homogéneo con respecto al poder para garantizar así la perpetuidad del sistema, tal y como sostiene Jackal, 2011.

Tras la muerte de Franco y la coronación de Juan Carlos I como rey de España, el poder gubernamental siguió en manos de Arias Navarro quien dimitió en julio de 1976. Por nombramiento del monarca, Adolfo Suárez (ex secretario general del Movimiento Nacional) pasó a ser presidente del Gobierno, y no fue hasta junio de 1977 cuando la población española votara a su presidente en los primeras elecciones democráticas desde 1936 en España. Pero, ¿qué comportamiento tuvo la prensa oficial con respecto al Gobierno Suárez en este período electoral teniendo en cuenta que hasta abril de 1977 había pertenecido al Movimiento Nacional?

Por un lado, la muerte de Franco y la consecuente caída del régimen, y por el otro el aumento de la presencia de ideologías de la oposición (socialismo, comunismo, etc.), acompañado de un clima de inestabilidad política agravado por el terrorismo provocaron un panorama controvertido en la prensa oficial. Gracias a esa inestabilidad, en esta prensa se dieron cita colaboradores de distintas ideologías (Martínez Fábregas, 2014), alejándose así de la función de propaganda del régimen que había tenido hasta entonces.

En esta línea, nuestra intención es, por una parte, analizar el proceder de estas cabeceras de titularidad estatal durante las elecciones legislativas, comprobando si seguían el proceder previsto en una cadena de prensa de este tipo. Y, por otro, desmitificarlas como órgano de propaganda del Gobierno durante este lapso de tiempo. Tesis ésta que ha sido tradicionalmente sostenida por Montabes Pereira (1989), quien afirma que la prensa oficial mantuvo su función propagandística con respecto al Gobierno. Por ello, el mencionado autor concluye que no evolucionó en este sentido durante este período. En la misma línea, Alférez (1986) sostiene que las ideologías de los trabajadores de la prensa oficial nunca llegaban a las páginas de la misma, que se esmeraba en ser un órgano de propaganda del Gobierno durante este período, o al menos durante el turno de Arias en el poder (Redero y García, 1991). Se garantizaba así, según los autores, el mantenimiento de un discurso único y unificado propio de los órganos de propaganda (Postoutenko, 2010) en el que no caben posicionamientos contrarios al poder (Pineda, 2006).

Sin embargo, Castro Torres (2010) reconoce la existencia de distintas ideologías dentro de la prensa oficial durante la transición, que le permitieron asumir su rol de agente político. Además, Peña Marín (1980) reconoce que, ya durante la dictadura, revistas como El Escorial (dirigida por Laín Entralgo y Dionisio Ridruejo) acogieron posicionamientos contrarios al régimen. Más allá va la profesora Meneses (2008), quien afirma que, durante la transición, la prensa oficial acogió distintos discursos en sus páginas.

Fruto de este profundo debate sobre el verdadero proceder de la prensa oficial con respecto al Gobierno durante la transición española surge nuestra pregunta de investigación: 
P1: ¿Continuaron estos diarios siendo un órgano de propaganda del gobierno durante las elecciones legislativas de 1977 ?

Partiendo de esta pregunta básica, nos proponemos como objetivos de investigación los siguientes:

- Demostrar la evolución del posicionamiento que los periódicos oficiales tuvieron con respecto al Gobierno durante este período y el abandono de su función propagandística.

- Demostrar la existencia de un discurso heterogéneo en la cadena y dentro de cada uno de los diarios fruto de la pluralidad ideológica que existía en sus redacciones.

- Demostrar la función de agente político del diario en el espectro periodístico español de la transición, adquirido a través del abandono de su función propagandística del Gobierno.

Para dar respuesta a esta pregunta alcanzando así los objetivos propuestos, partimos de las siguientes hipótesis formuladas a partir del método hipotético- deductivo:

(H1): La prensa oficial no mantiene un discurso único y unificado con respecto al Gobierno durante este momento histórico.

(H2): Cada diario analizado mantuvo un discurso heterogéneo con respecto al Gobierno en este hito histórico, fruto de los diferentes posicionamientos sostenidos por el editorialismo, el columnismo y el articulismo de cada uno de ellos.

(H3): En este sentido, la prensa oficial acoge críticas contra el Gobierno durante los comicios electorales abandonando su función propagandística y asumiendo su rol de agente político activo.

Una vez formulados las hipótesis y los objetivos de la presente investigación, procedemos a explicar la metodología que hemos aplicado a nuestro objeto de estudio.

\section{Metodología}

Para la elaboración de este trabajo, hemos utilizado en primer lugar, el estudio basado en la biografía técnica que propone Casasús (1985) sobre el diario seleccionado. La ficha hemerográfica del periódico (re-elaborada sobre una propuesta del doctor Marcel Mauri, en su tesis doctoral, 2010. Véase ficha de análisis hemerográfico en el apartado Anexos ) nos ha permitido realizar un estudio biográfico de las cabeceras seleccionadas, además de averiguar la inclinación ideológica de los firmantes en este diario durante la transición, lo cual constituye una de las principales líneas de este trabajo.

Una vez analizadas hemerográficamente, procedemos a seleccionar el corpus de trabajo que hemos analizado en la presente investigación. Para ello, hemos seguido las fases planteadas por Winner y Dominick (2000: 141-144). En base a esto, hemos seleccionado dos cabeceras de ámbito nacional, una regional y cuatro locales, que han sido agrupadas de la siguiente forma:

$1^{\circ}$. Referentes centrales: alude a los diarios de difusión nacional editados en Madrid. 
- Arriba: Periódico de referencia del Movimiento. De ámbito nacional y con 20.000 ejemplares de difusión diaria durante el período de estudio, fue el órgano de propaganda de Falange Española desde su fundación en 1935, y la "voz" de Franco según Jaime D'Amico (2008).

- Pueblo: diario vespertino de Madrid, propiedad del Sindicato Vertical. También de ámbito nacional, y con una difusión de 179.000 ejemplares diarios entre 1975 y 1978, tuvo una plantilla de 650 periodistas durante este período.

A pesar de la diferencia numérica existente entre la difusión de Arriba y Pueblo, se han seleccionado estos dos diarios por ser referencia del régimen en el ámbito nacional.

$2^{\circ}$. Referentes de las nacionalidades: alude a los diarios editados en los territorios con tradición política nacionalista.

- Solidaridad Nacional: diario matutino de Barcelona que procedía de Solidaridad Obrera, periódico cenetista incautado por las tropas franquistas en 1939. Tenía una difusión de 5.400 ejemplares diarios en este período.

- El Pueblo Gallego, de Vigo: diario de la mañana que nació en 1924 con un claro posicionamiento regionalista. Se editó en gallego hasta 1936. Alcanzó una difusión de 5.100 ejemplares diarios durante la transición.

- Hierro, de Bilbao: periódico de la tarde, que ocupaba las instalaciones del socialista El Liberal, dirigido por Dionisio Ridruejo, e incautado por las tropas nacionales tras la toma de Bilbao. Tenía una difusión de 5.200 ejemplares diarios durante la transición.

$3^{\circ}$. Referentes regionales: diarios publicados en Andalucía, cuarta comunidad en acceder a la autonomía.

- Sur, de Málaga: Referente regional de la cadena de Prensa del Movimiento Nacional en Andalucía. Fue el único periódico rentable de la cadena. Según Ruiz Romero (1998), tuvo una tirada de 22.659 ejemplares diarios.

- Odiel, de Huelva: Diario de la mañana, fundado en 1935 por el PRC (Partido Republicano Conservador). Se trataba del segundo diario con menor difusión de la región (5755 ejemplares diarios).

Sobre este corpus, procedemos al análisis de contenido cualitativo explícito, que consiste en un estudio sistemático de una muestra seleccionada de forma objetiva haciendo inferencias válidas y confiables en función del contexto en el que los textos fueron producidos (Krippendorff, 1982). Para su análisis, nos hemos decantado por centrarnos en el contenido explícito o manifiesto que, según Landry (1991: 341), "remite a lo que es dicho o escrito explícitamente [...], vehicula la totalidad del significado".

Una vez seleccionado el método, para su aplicación es necesario recopilar la muestra siguiendo los principios que nos propone Krippendorf (1982). Es por ello que, para la selección del contenido referente a este momento histórico, se ha atendido a lo que sostiene Paul Lazarsfeld (1953) en su análisis sobre las presidenciales americanas. La 
técnica que utilizó para su estudio fue la encuesta mensual a diferentes grupos representativos en diferentes momentos. Es decir, la población (600 personas) fue entrevistada siete veces en distintos puntos de la campaña electoral. La última vez fue la semana antes de las elecciones. El autor concluyó entonces que los grupos de electores se homogeneizan a medida que se acerca el día de las elecciones y que, en la última semana de la campaña, los propagandistas dirigieron sus esfuerzos a los 'indecisos' (1953: 407). Por esta razón, en el presente estudio se ha seleccionado la semana previa a los comicios y los tres días posteriores a los mismos (es decir, desde el 8 hasta el 18 de junio), ya que no sería hasta el 16 de junio por la tarde cuando se lanzaran los resultados definitivos de los comicios informando de la victoria de la UCD.

Seleccionado el período de estudio, procedemos a la selección de las unidades de análisis. Atendiendo a lo sostenido por Berelson (1952), quien las considera como segmentos del contenido caracterizados para su ubicación dentro de categorías, hemos optado por los ítems (unidad total de material simbólico), porque el método de esta investigación se ha aplicado a textos periodísticos, es decir, "una unidad total de material simbólico".

Teniendo en cuenta que nuestras hipótesis persiguen demostrar la existencia de distintos puntos de vista ideológicos dentro de estos diarios, hemos optado por el análisis de los editoriales, los artículos y las columnas de opinión. Pero, ¿por qué estos géneros y no otros?

En primer lugar, hemos seleccionado los editoriales porque es el texto de opinión que goza de menor libertad a la hora de registrar tanto su lenguaje como su técnica y su estructura; es decir, "es un texto generalmente uniforme y de criterios más o menos fijos" (López Hidalgo, 2012: 35).

Por otro lado, para León Gross (1996: 145), el estudio del columnismo y el articulismo como fuente para analizar la prensa, "ha sido poco prolífico, hasta fechas recientes, por no estar encuadrado claramente en alguno de estos dos ámbitos (periodismo o literatura)". Coincide con Villalobos (2010) quien afirma que, tradicionalmente, se ha estudiado la línea editorial de los periódicos para conocer el verdadero proceder del mismo con respecto a un tema concreto, ignorando el papel del columnismo y el articulismo en el asentamiento de un estado de opinión.

Sin embargo, para Martínez Albertos (1992: 382), las columnas constituyen unos espacios de tema libre destinados a escritores de prestigio y fama, con la única condición de que firmen sus textos. En este sentido, las columnas comparten el carácter de 'tema libre' con los artículos de opinión, de los que Martín Vivaldi (1993: 176) dice que son escritos "de muy vario y amplio contenido, de varia y muy diversa forma, en el que interpreta, valora o explica un hecho o una idea actuales de especial trascendencia, según la convicción del articulista". Coincide con López Hidalgo (2012), quien asegura que las diferencias existentes entre lo sostenido por los editoriales y los artículos y las columnas de opinión pueden ser muy considerables, contradiciendo así a Abril Vargas (1999) quien afirma que estas diferencias no son más que meros matices.

Además, no sólo existen diferencias entre editoriales y columnas y artículos, sino también entre estos dos últimos. Así, para Martín Vivaldi (1993), el columnista es un periodista que dispone de un espacio concreto y de forma asidua en el periódico, mien- 
tras que el articulista es un colaborador esporádico y experto en el tema concreto sobre el que escribe. Y esto a su vez contribuye a la presencia de distintos posicionamientos, al no tener relación de obediencia con respecto al medio (Pineda, 2006), erigiéndose así columnismo y articulismo de opinión en los garantes de la pluralidad de opinión en el diario (Borrat, 1989).

Tomando como referencias estas afirmaciones, la confluencia de distintos discursos sobre un mismo tema en el periódico puede suponer la ausencia de un discurso único y unificado dentro del mismo, al no seguir las columnas y los artículos los dictados del editorial. Y, por otro lado, atendiendo a la libertad para la selección del tema y el posicionamiento ideológico que rigen las opiniones de columnistas y articulistas (López Hidalgo, 2012), esta realidad podría suponer la confluencia de distintos posicionamientos con respecto al Gobierno por parte de los periódicos analizados. Este hecho invalidaría a estos diarios como órgano de propaganda del Gobierno durante las elecciones legislativas, tal y como sostenían Alférez (1986), Montabes Pereira (1989) y Redero y García (1991).

De esta forma y una vez definidas las unidades de análisis, que nos dan una muestra total de 226 piezas, hemos de diseñar las categorías para la validación o refutación de nuestras hipótesis.

Según Berelson (1952), por categoría debe entenderse un concepto genérico que agrupa las proposiciones de varias unidades de registro. Coincide con Krippendorff (1982) en afirmar que las categorías son los niveles en los que se clasifican las unidades de análisis. Y de los posibles tipos que propone el autor, nosotros nos hemos centrado en la tipología de dirección, encaminada a determinar la validez de nuestras hipótesis. Por lo tanto, las categorías construidas para el presente trabajo de acuerdo con nuestras hipótesis son:

1. Posicionamiento Positivo (+): apoyo explícito al Gobierno.

2. Posicionamiento Neutro (0): Sin enjuiciar la gestión gubernamental.

3. Posicionamiento Negativo (-): críticas a la labor del Ejecutivo.

Y una vez expuesta la metodología procedemos a la presentación de resultados y la discusión del presente trabajo.

\section{Resultados y discusión}

En este apartado mostraremos en primer lugar los resultados del análisis cuantitativo que demuestran la existencia de diferentes posicionamientos no solo dentro de la cadena sino también en el discurso interno de cada uno de los diarios. Y, posteriormente, expondremos los matices que cada uno de los textos de opinión publicados en cada uno de los periódicos aportaba sobre los posicionamientos (positivo, neutro o negativo) con respecto al Gobierno y su proceder, constituyendo estos los datos cualitativos de la presente investigación. A través de ellos, podremos analizar en profundidad la plasmación de las distintas corrientes ideológicas confluyentes en los textos de opinión de la prensa oficial en su páginas de opinión, a la vez que establecer un mapa fiable de la conducta de estos con respecto al Gobierno durante las elecciones legislativas, lo que nos permitirá el alcance o no de nuestros objetivos. 
En este sentido, la tabla que se muestra a continuación muestra los datos cuantitativos referentes al posicionamiento de las unidades de análisis con respecto al Gobierno durante las elecciones legislativas.

Tabla 1: Posicionamiento de los textos de opinión con respecto al Gobierno durante las elecciones legislativas (15 de junio de 1977). Las siglas 'Ed' se refiere a los editoriales y 'PO' a las piezas de opinión firmada. Fuente: elaboración propia

\begin{tabular}{|c|c|c|c|c|c|c|c|c|c|c|c|c|c|c|}
\hline \multirow[t]{3}{*}{ Pos. } & \multicolumn{4}{|c|}{ Diarios centrales } & \multicolumn{6}{|c|}{ Diarios de nacionalidades } & \multicolumn{4}{|c|}{ Diarios regionales } \\
\hline & \multicolumn{2}{|c|}{ Arriba } & \multicolumn{2}{|c|}{ Pueblo } & \multicolumn{2}{|c|}{$\begin{array}{l}\text { Solidaridad } \\
\text { Nacional }\end{array}$} & \multicolumn{2}{|c|}{$\begin{array}{l}\text { El Pueblo } \\
\text { Gallego }\end{array}$} & \multicolumn{2}{|l|}{ Hierro } & \multicolumn{2}{|l|}{ Sur } & \multicolumn{2}{|l|}{ Odiel } \\
\hline & ED & PO & ED & PO & ED & PO & ED & PO & ED & PO & ED & PO & ED & PO \\
\hline+ & $72,7 \%$ & $60,9 \%$ & $75 \%$ & $18,7 \%$ & $75 \%$ & $16,6 \%$ & $33,3 \%$ & $18,3 \%$ & $33,3 \%$ & $0 \%$ & $0 \%$ & $12,5 \%$ & $33,3 \%$ & $50 \%$ \\
\hline 0 & $27,3 \%$ & $26,2 \%$ & $12,5 \%$ & $42,7 \%$ & $0 \%$ & $83,3 \%$ & $66,6 \%$ & $73,3 \%$ & $66,6 \%$ & $50 \%$ & $100 \%$ & $45 \%$ & $66,6 \%$ & $50 \%$ \\
\hline- & $0 \%$ & $12,9 \%$ & $12,5 \%$ & $38,5 \%$ & $25 \%$ & $0 \%$ & $0 \%$ & $8,40 \%$ & $0 \%$ & $50 \%$ & $0 \%$ & $42,5 \%$ & $0 \%$ & $0 \%$ \\
\hline
\end{tabular}

Como se puede observar en la tabla anterior, la celebración de las elecciones del 15 de junio tuvo una acogida mayormente favorable al Gobierno en la línea editorial de Arriba, Pueblo y Solidaridad Nacional y, sobre todo neutro en El Pueblo Gallego, Hierro, Sur y Odiel. Únicamente se aprecia cierta crítica en el sentir oficial de Pueblo y Solidaridad Nacional.

Los datos relativos a las piezas de opinión firmada desvelan posturas mucho más encontradas. Así, destaca el apoyo al Ejecutivo en Arriba, donde la crítica y la neutralidad tienen valores muy discretos, mientras que las posturas neutras destacan en el resto de diarios, a excepción de Odiel, donde neutro y positivo tienen el mismo valor. Asimismo, las críticas al Ejecutivo en las piezas de opinión destacan en Pueblo, Hierro y Sur, y en menor medida en Arriba y El Pueblo Gallego, no registrándose datos en el caso de los referentes catalán y onubense.

Con esto se valida, al menos a nivel cuantitativo, nuestra primera hipótesis que asumía la presencia de diferentes posicionamientos en los diarios analizados. De esta forma, constatamos que dentro de la prensa oficial no existió un discurso único y unificado con respecto al Gobierno durante las elecciones legislativas de junio de 1977. Se valida asimismo lo sostenido por Borrat (1989) y Villalobos (2010) quienes consideraban a las piezas de opinión firmada como los garantes de la pluralidad de opinión en un diario. Y es que, como podemos observar en la tabla, mientras en la línea editorial de los diarios analizados, el posicionamiento positivo era mucho mayor, en las piezas de opinión, las posturas neutra y negativa tienen un mayor peso.

Por lo tanto, la línea editorial de los diarios Arriba, Pueblo, Solidaridad Nacional $^{2}$, El Pueblo Gallego, Hierro y Sur apostaron en estos comicios por la moderación de $\mathrm{UCD}^{3}$ avalada por la figura del Rey Juan Carlos $\mathrm{I}^{4}$, exigiendo un espíritu concilia-

2 Solidaridad Nacional, 16/06/1977, p. 3: Triunfó la moderación.

3 Pueblo, 09/06/1977, p. 3: No todo tan negro y Pueblo, 13/06/1977, p. 3, Editorial: "Lo que tenía que hacer...”.

4 Arriba, 08/06/1977, p. 7: *Ponderar la moderación y Arriba, 11/06/1977, p. 7: *Se acertó en lo principal. 
dor de los extremos ideológico-políticos ${ }^{5}$ existentes en España y agradeciendo la pluralidad política concurrente a las primeras elecciones democráticas ${ }^{6}$.

En cuanto al posicionamiento neutro, en el sentir oficial de Solidaridad Nacional y Odiel destacaron la preocupación de la población por los problemas que sufría el país ${ }^{7}$ y El Pueblo Gallego hizo hincapié en que, a través del ejercicio del derecho a voto en las elecciones, el pueblo había recuperado su soberanía ${ }^{8}$.

Finalmente y, en sentido opuesto al Gobierno, el diario sindicalista exigía al vencedor de las elecciones que abandonara la tónica de tráfico de influencias que se había mantenido hasta entonces en la formación del Gobierno español, acusando a Suárez de haber sido un presidente escogido a dedo por el Rey, y que a su vez había escogido de la misma forma a su gabinete, a la vez que Hierro criticaba los incidentes registrados en la emisión del voto y los achacaba a la falta de información que el poder había ofrecido a los electores ${ }^{10}$.

De esta forma, Arriba, Pueblo, El Pueblo Gallego y Hierro apostaron por UCD como partido victorioso en las elecciones, destacando todos ellos la labor democratizadora del Gobierno. En un tono más neutro, sin posicionarse abiertamente, Sur pedía la moderación en el voto, lo que indirectamente estaba asociado a UCD. Y, finalmente, Pueblo y Solidaridad Nacional acogían discursos encontrados dentro de su línea editorial, y si bien apostaban por la moderación de UCD como victorioso en las elecciones, le criticaban su proceder desde la propia formación del Gobierno y se referían a las elecciones en contraposición con los problemas de los que adolecía el país y que preocupaban a los ciudadanos españoles.

Comprobamos así que ni siquiera la línea editorial de estos medios seguía un único proceder, y que dentro del sentir oficial de cada diario se daban cita distintos posicionamientos con respecto al Gobierno, fruto de la pluralidad ideológica existente en los consejos editoriales de estas cabeceras. No es de extrañar cuando, por ejemplo, socialismo y ucedismo estaban representados en la misma medida en los consejos editoriales de estos diarios, seguidos en menor medida por el falangismo, franquismo, comunismo, sindicatos (UGT, CCOO, y otros) y Opus Dei, tal y como se puede comprobar en el gráfico siguiente:

${ }^{5}$ Arriba, 15/06/1977, p. 7: *El espíritu del 15 de junio.

Sur, 15/06/1977, p. 1: El espiritu del 15 de junio.

Odiel, 12/06/1977, p. 2: Programas y actitudes electorales.

8 Solidaridad Nacional, 16/06/1977, p. 3: El pueblo ha recuperado la soberanía.

9 Pueblo, 17/06/1977, p. 3: Reforma, política y burocracia.

${ }^{10}$ Hierro, 16/06/1977, p. 3: Respeto democrático. 
Gráfico 1: Confluencia de ideologías ${ }^{11}$ en los consejos editoriales de los diarios oficiales durante la transición.

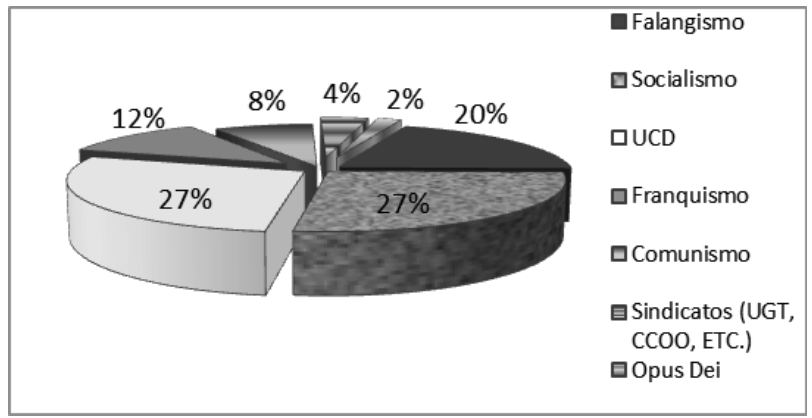

Fuente: Martínez Fábregas, Jezabel (2014): La imagen del Gobierno en la prensa oficial durante la transición española. Análisis comparativo de Arriba, Pueblo, Solidaridad Nacional, El Pueblo Gallego, Hierro, Sur y Odiel. Universidad de Sevilla

Por otro lado, esta pluralidad de opiniones constatada en el consejo editorial de los diarios analizados es aún más visible en el caso de las opiniones firmadas, como se puede observar en la tabla 1.

De esta forma, y en lo que a los referentes centrales se refiere, en Arriba se instaba a votar a los conservadores, a la vez que se criticaba de forma indirecta al Gobierno ${ }^{12}$ por la legalización del PCE, destacando que, aunque el Ejecutivo fuera el mismo tras el 15 de junio, ahora el Parlamento era de representación popular ${ }^{13}$. Por ello, José Cavero argumenta que no es al Gobierno Suárez al que se le debe la jornada democrática del 15 de junio, sino a la presión y la movilización popular ${ }^{14}$.

Más crítico fue Evaristo Acevedo, que arremetió duramente contra el Gabinete Suárez en Pueblo (siguiendo la línea editorial de Hierro) por no haber explicado a la población el proceso de emisión del voto, sobre todo teniendo en cuenta que eran las primeras elecciones que se celebraban en el país desde 1936. Lo responsabilizó además de los errores que cometieron los electores en su voto ${ }^{15}$ en dichos comicios y que provocaron la nulidad de los mismos.

${ }^{11}$ Las ideologías de cada uno de los conformantes de los consejos editoriales de estos diarios ha sido averiguada atendiendo a las siguientes fuentes: 1) registros oficiales de militancia en partidos políticos; 2) autoproclamación en público a través de una entrevista concedida a un medio de comunicación o en sus propios textos de opinión en los que confiesan su inclinación ideológica. Para comprobar la inclinación ideológica de cada miembro de los consejos editoriales de los diarios analizados, se puede acceder a la Tesis Doctoral de Martínez Fábregas, Jezabel (2014): La imagen del Gobierno en la prensa oficial durante la transición española (1975-1978). Análisis comparativo de Arriba, Pueblo, Solidaridad Nacional, El Pueblo Gallego, Hierro, Sur y Odiel. Universidad de Sevilla.

${ }_{12}$ Arriba, 14/06/1977, p. 12, Lavern: Dicho y oído.

${ }^{13}$ Arriba, 15/06/1977, p. 5, Antonio Salcedo: La Situación.

${ }^{14}$ Arriba, 15/06/1977, p. 6, José Cavero: Día uno de la democràcia.

${ }^{15}$ Pueblo, 09/06/1977, p. 11, Evaristo Acevedo: Por la sonrisa hacia el futuro. El detalle. 
En este tono de crítica, Eduardo G. Rico acusó al Gobierno de mantener a políticos del anterior régimen en sus filas ${ }^{16}$. Por su parte, José Elizalde, quien se autocalifica en el texto como anarcosindicalista, criticó la campaña electoral y el sistema de autogestión del Gabinete Suárez, animando a la abstención en las elecciones ${ }^{17}$.

En el extremo opuesto, Carlos Iglesias Selgas apostó por la victoria del centro-derecha en la que UCD y AP tendrían que pactar para gobernar. Según él, esto daría tiempo a la izquierda para organizarse y acceder al Ejecutivo en los próximos comicios, trayendo así el modelo democrático europeo a España ${ }^{18}$. Más duro se mostró Emilio Aguilar quien acusó al Gobierno de engañar al electorado durante la campaña, prometiendo soluciones a los problemas del país sin decirles que éstas supondrían sacrificios por parte de la población ${ }^{19}$.

Y, tras la victoria de la UCD en las elecciones legislativas, José Bugeda criticó al Gabinete Suárez por mantener un clima de desconfianza en la población basado en las mentiras de la campaña electoral ${ }^{20}$. En la misma línea, Pilar Narvión se centró en destacar los problemas económicos que sufría el país, a la vez que exigía al Parlamento la unificación de fuerzas para solucionarlo, aunque responsabilizaba mayoritariamente al Gobierno 'electo'21.

Los diarios de las nacionalidades, por su parte, también acogieron diferentes posicionamientos con respecto al Ejecutivo. De esta forma, Aristarco destacó en el referente catalán los problemas sociales (siguiendo así lo marcado desde la línea editorial) y pedía reflexión por parte de la población en el momento de ejercer su derecho a voto, dando libertad para votar a quien quisiera ${ }^{22}$. Por su parte, Demetrio Castro Villacañas se centró en criticar la espectacularización que rodeaba a los mítines de los candidatos $^{23}$. Finalmente, El Discreto analizó el resultado de las elecciones, ignorando la victoria de $\mathrm{UCD}^{24}$. Igual proceder adoptó Aristarco que ignoró al Ejecutivo y se centró en los resultados obtenidos por PSOE y PCE, destacando la presencia del primero en el territorio catalán y la previsible fuerza que alcanzaría en los próximos comicios municipales ${ }^{25}$.

En el extremo opuesto se situó De Almazán, quien destacó en El Pueblo Gallego que el Gobierno Suárez contaba con la aprobación de los EEUU reforzando así la idea de que la victoria de UCD en las elecciones era un triunfo para el país ${ }^{26}$.

\footnotetext{
${ }^{16}$ Pueblo, 11/04/1977, p. 9, Eduardo G. Rico: No es la izquierda sino el centro.

${ }^{17}$ Pueblo, 09/06/1977, P. 12, José Elizalde: La cuadratura del círculo electoral.

${ }_{18}$ Pueblo, 13/06/1977, p. 9, Carlos Iglesias Selgas: La significación del próximo gobierno.

${ }_{19}$ Pueblo, 15/06/1977, p. 9, Emilio Aguilar: Lo que falta en los programas.

${ }^{20}$ Pueblo, 16/06/1977, p. 19, José Bugeda: Bien ¿y ahora qué?

${ }^{21}$ Pueblo, 16/06/1977, p. 19, Pilar Narvion: A trabajar todos.

${ }_{22}$ Solidaridad Nacional, 12/06/1977, p. 2. Aristarco, Catalejo, Los miedos por la ventana.

${ }^{23}$ Solidaridad Nacional, 12/06/1977, p. 2. Demetrio Castro Villacañas, Al margen. Las atracciones.

${ }^{24}$ Solidaridad Nacional, 19/06/1977, p. 3. El Discreto, Manual de Perplejos. El espectro de las elecciones.

${ }_{25}$ Solidaridad Nacional, 19/06/1977, p. 3. Aristarco, Sorpresas relativas.

${ }^{26}$ El Pueblo Gallego, 18/06/1977, p. 3. Juan de Almazán. La jornada.
} 
Y en el vaivén de críticas y halagos al Gobierno, Salcedo consideró a Suárez como el gestor del milagro por haber llevado a España a decidir quién quería que la gobernara $^{27}$. Coincidió con Cavero, que también destacó que el país le debía a Suárez el cumplimiento del sueño de poder votar ${ }^{28}$. Por el contrario, a una semana de los comicios, Palomino criticó al Ejecutivo por haber hecho un ingente gasto en cosas innecesarias en tiempos de crisis sin control alguno ${ }^{29}$.

Más allá de la crítica fue Lawrence que acusó al Gobierno de no haber escuchado a la oposición cuando solicitó la estipulación de unas normas técnicas ${ }^{30}$ para evitar la manipulación de las encuestas ${ }^{31}$. Por su parte, Aller se posicionó en tono neutro, incitando al pueblo a que se pronunciase en los comicios haciendo uso de sus derechos y sin decantarse por ninguna opción ${ }^{32}$.

En una línea más crítica se manifestó Cavero en Hierro, que arremetió contra el «presidente candidato» por presentarse a las elecciones sin disolver las Cortes ni abandonar su estatus de Jefe de Gobierno ${ }^{33}$. En Sur, Marco criticó la cercanía del Gobierno a la derecha e incitó a la opinión pública a no votar a aquellos partidos que tenían estas relaciones ${ }^{34}$. José Cavero además, exigía al Gobierno que sentara las bases que posibilitaran la imparcialidad electoral, ya que a su juicio, el ser presidente-candidato le otorgaba gran ventaja sobre el resto de candidatos a los comicios ${ }^{35}$.

$\mathrm{Y}$ en el mismo diario Antonio Salcedo criticó las mentiras lanzadas por los candidatos durante la campaña electoral, otorgándole la victoria en las urnas a UCD, y afirmando que el precio de esas mentiras políticas lo pagarían los españoles 'cuando pasaran las elecciones' ${ }^{36}$. En la misma línea, José Luís Casas, un preso del régimen franquista, rompió una lanza a favor del Gobierno Suárez, afirmando que éste había hecho más por la democracia que ningún otro ${ }^{37}$.

Y en la misma tónica de las opiniones de Casas en el regional, en el local onubense, Antonio Salcedo destacó el talante de Suárez como candidato y remarcó que su espíritu de moderación era la mejor vía para el advenimiento de la democracia ${ }^{38}$.

De esta forma, podemos afirmar que los textos de opinión firmada publicados en la prensa oficial no solo no mantuvieron un discurso uniforme, en cadena, y a favor del Gobierno como sostenían Montabes Pereira (1989), y Alférez (1986), sino que ni siquiera cada uno de los diarios analizados mantenía un discurso único, fruto de la pluralidad ideológica existente en las redacciones (Martínez Fábregas, 2014).

\footnotetext{
${ }^{27}$ El Pueblo Gallego,08/06/1977, p. 3. Antonio Salcedo, Una cortesía mínima.

${ }^{28}$ El Pueblo Gallego, 10/06/1977, p. 3. José Cavero, El Presidente Candidato.

${ }^{29}$ El Pueblo Gallego, 08/06/1977, p. 3. Ángel Palomino, $<<$ España impresa $>>$.

${ }^{30}$ Hierro, 16/06/1977, p. 7. Lawrence, La manipulación por las encuestas.

${ }^{31}$ El Pueblo Gallego, 15/06/1977, p. 3. Lawrence, La manipulación de las encuestas.

${ }^{32}$ El Pueblo Gallego, 15/06/1977, p. 3. Ramón María Aller: Participación ciudadana.

${ }^{33}$ Hierro, 10/06/1977, p. 8. José Cavero: El presidente candidato.

${ }^{34}$ Sur, 10/06/1977, p. 7. Marco: EN VÍSPERA DE LAS ELECCIONES, Precisando.

${ }_{35}$ Sur, 10/06/1977, p. 33. José Cavero: El presidente candidato.

${ }^{36}$ Sur, 10/06/1977, p. 33, Antonio Salcedo: LA SITUACIÓN, Programas.

${ }^{37}$ Sur, 11/06/1977, p. 30, José Luis Casas: Más difícil todavía.

${ }^{38}$ Odiel, 12/06/1977, Antonio Salcedo: La situación, Un talante.
} 
Esto permitió la presencia de discursos contrarios y favorables al Gobierno, así como neutros, rompiendo la estructura de cualquier modelo de propaganda, ya que según Pineda (2006) un medio propagandístico no puede asumir discursos contrarios al poder, y validando nuestra primera hipótesis. Y precisamente este alejamiento de su función propagandística permitió a esta prensa la asunción de su rol de agente político.

De esta forma, la confluencia de distintas ideologías en los consejos editoriales, unida la libertad de escoger tema y expresar su opinión, que Borrat (1989) y Martín Vivaldi (1986) le reconocen al articulismo y al columnismo, permitió que los diarios oficiales analizados no mantuvieran un discurso único y unificado con respecto al Gobierno. Por lo tanto, estos periódicos, al formar parte de la cadena de periódicos oficiales, no mantuvieron un único proceder con respecto al poder del Ejecutivo, tal y como se prevé en la prensa de este tipo, según Pineda (2006).

Así, la validación de nuestra primera hipótesis (párrafo anterior) nos lleva a validar nuestra segunda, demostrando que la pluralidad ideológica existente en las redacciones de las cabeceras analizadas se tradujo en la convivencia de distintos discursos dentro de las páginas de opinión de los periódicos oficiales analizados. Además, al convivir distintas ideologías políticas en los consejos editoriales y, gracias a la libertad para la expresión de sus opiniones de columnistas y articulistas al no contar en una gran proporción con una relación contractual con los diarios para los que escribían (Martínez Fábregas, 2014), ni siquiera se ha constatado la existencia de un único discurso dentro de cada diario analizado.

Con esto, comprobamos que las diferentes ideologías que convivían en estos diarios trascendieron del imaginario colectivo de cada autor hasta las páginas de opinión de los diarios, convirtiéndolas en un espacio de debate político. Asimismo, queda constancia de que la diversidad ideológica existente en cada uno de estos periódicos propició que las páginas de opinión de estas cabeceras acogieran puntos de vista muy diversos y a veces contradictorios entre sí. Esta afirmación contradice lo sostenido por Alférez (1986), quien afirmaba que a pesar de que tuvieran ideología distintas, los firmantes de textos de opinión en la prensa oficial nunca expresaron en sus páginas puntos de vista distintos al oficial, es decir, al que interesaba y emanaba del Gobierno.

En este sentido, los diarios analizados abandonaron su función propagandística con respecto al Gobierno y su gestión, al contrario de lo que sostenía Alférez (1986), y comenzaron a asumir su rol de agente político dentro del conocido parlamento de papel, validándose así la tercera hipótesis de esta investigación.

Y una vez expuestos los resultados y la discusión del presente trabajo, procedemos a la exposición de las conclusiones.

\section{Conclusiones}

La prensa oficial no fue un órgano de propaganda del Gobierno durante la transición. Lo demuestra la confluencia de distintos discursos dentro de sus páginas de opinión, lo que permite a su vez una pluralidad de puntos de vista sobre el Ejecutivo y su gestión que demuestran que acogió, durante este momento histórico de especial relevancia para el futuro político del país, una considerable pluralidad de opinión imprevisible en una prensa de este tipo -que había estado al servicio del poder como órgano de propaganda- en un estado aún autoritario, y en transición hacia la democracia. 
Por otro lado, los datos obtenidos demuestran la existencia de diversos discursos dentro las páginas de opinión de estos diarios gracias a los distintos puntos de vista que, sobre la realidad, aportan editoriales, artículos y columnas de opinión. Tal y como se ha constatado en esta investigación, estas tres unidades de análisis no mantienen un discurso único y unificado con respecto al Gobierno sino que, por el contrario, reflejan distintos posicionamientos, a veces, contrarios entre sí dentro de las páginas de opinión. De esta forma, hemos constatado que los artículos y las columnas de opinión publicados en estos periódicos durante las elecciones legislativas de 1977 se erigieron en garantes de la pluralidad de opinión no sólo a nivel de cadena sino también dentro de cada uno de los diarios analizados. Esto supone que no difundieron un discurso unificado con respecto al Ejecutivo, lo que es un requisito sine qua non para ser órgano de propaganda del mismo.

Asimismo, durante las elecciones legislativas, los diarios analizados emprendieron duras críticas contra el Gobierno por la gestión que estaba llevando a cabo durante las mismas. Se le acusó de controlar los resultados electorales, intentando soslayar el peso de la oposición. Así la prensa oficial fue abandonando su función propagandística con respecto al Gobierno y su gestión de la transición, comenzando a asumir su rol de agente político.

Finalmente, se ha constatado que no existió ni siquiera un discurso único y unificado dentro de cada uno de los diarios analizados, ya que mientras el posicionamiento de los editoriales es (excepto en Pueblo y Solidaridad Nacional) muy favorable al Gobierno, en las piezas de opinión firmada encontramos la verdadera pluralidad discursiva de estos diarios que terminan convirtiéndose en los verdaderos garantes de la pluralidad de opinión en estos periódicos.

Para concluir podemos afirmar que la prensa oficial no fue un órgano de propaganda del Gobierno Suárez durante las elecciones legislativas de 1977, ya que acogió duras críticas contra el Ejecutivo y su gestión. Éstas eran fruto de los diferentes posicionamientos que editorialistas, columnistas y articulistas tenían, lo que permitía la convivencia de una considerable pluralidad ideológica en estos diarios. Asimismo, fue esta pluralidad ideológica la que permitió que estos periódicos abandonaran su función propagandística y asumieran su rol de agente político.

\section{Anexo}

Tabla 1: Modelo de Ficha hemerográfica ${ }^{39}$

\begin{tabular}{|l|l|l|}
\hline \multicolumn{3}{|c|}{ Modelo de Ficha hemerográfica } \\
\hline Datos registrales & Datos formales y de contenido & \multicolumn{1}{c|}{ Datos periodisticos } \\
\hline Nombre de la publicación & Formato y presentación & Consejo editorial \\
\hline & & $\begin{array}{c}\text { Comité de redacción: } \\
\text { 1. Editorialistas }\end{array}$ \\
Subtítulo, lema y otros elementos de la cabecera & Medidas: base x altura & 2. Columnistas \\
& & 3. Articulistas \\
& & 4. Redactores \\
\hline
\end{tabular}

${ }^{39}$ Los parámetros destacados en cursiva son los ítems que hemos introducido en la ficha de análisis propuesta por el profesor Mauri (2010: 85-89) para la presente tesis. 


\begin{tabular}{|c|c|}
\hline Lugar de edición (ciudad, provincia y comarca) & $\begin{array}{l}\text { Técnicas de impresión } \\
\text { 1. Tipografía } \\
\text { 2. Color } \\
\text { 3. № habitual de páginas } \\
\text { 4. № habitual de columnas }\end{array}$ \\
\hline Ámbito & $\begin{array}{l}\text { Agencias de información: } \\
\text { 1. Nacionales, propias o externas } \\
\text { 2. Internacionales }\end{array}$ \\
\hline Días y horarios de aparición & Orientación política \\
\hline Lengua o lenguas & \\
\hline Porcentaje de uso & \\
\hline Fecha de primer número & \\
\hline Fecha del último número & \\
\hline Precio de venta & \\
\hline Precio de suscripción & \\
\hline Ediciones extraordinarias & \\
\hline Incidencias, suspensiones, multas, etc. & \\
\hline Colecciones conservadas & \\
\hline Archivos, bibliotecas y hemerotecas digitales & \\
\hline Referencias externas en prensa & \\
\hline Indicación de números conservados & \\
\hline Dirección de la redacción & \\
\hline Dirección de la administración & \\
\hline Dirección del impresor & \\
\hline
\end{tabular}

Fuente: Elaboración propia sobre la ficha propuesta por Mauri (2010: 85-89). Los parámetros destacados en cursiva son los ítems que hemos introducido en la ficha de análisis propuesta por el profesor Mauri (2010: 85-89) para la presente tesis

\section{Referencias bibliográficas}

ALFÉREZ, Antonio (1986): Cuarto poder en España: la prensa desde la Ley Fraga 1966. Espulgues de Llobregat, Plaza \& Janés.

CASTRO TORRES, Carmen (2010): La Prensa en la transición española 1966/1982. Madrid, Alianza Ensayo.

BARRERA, Carlos (1995): Sin mordaza: veinte años de prensa en democracia. Madrid, Temas de hoy.

BERELSON, Bernard (1952): Content Analysis En: Handbook of Social Psychology, Vol I. New York, Free Press.

BORRAT, Héctor (1989): El periódico actor político. Barcelona, Gustavo Gili.

FUSI, Juan Pablo (1996): “El desarrollo autonómico”. En TUSELL, Javier y SOTO, Álvaro: Historia de Transición, 1975-1986. Madrid, Alianza Universidad.

GUILLAMET, Jaume (2002). "Factores de progreso y atraso en la evolución histórica del periodismo. El franquismo". En GARCIA GALINDO, Juan Antonio; GUTIÉRREZ LOZANO, Juan Francisco; SÁNCHEZ ALARCÓN, Inmaculada (eds.): La comunicación social durante el franquismo. Málaga, Centro de Ediciones de la Diputación Provincial de Málaga.

KRIPPENDORFF, Klaus (1982): Content Analysis; An Introduction to its Methodology. Beverly Hills, CA, Sage.

KRIPPENDORFF, Klaus (2012): Content Analysis; An Introduction to its Methodology, 3rd Edition. Thousand Oaks, CA, Sage. 
LANDRY, Réjean (1998): “L'analyse de contenu". Recherche Sociale. De la problemátique à la collecte des données. Benoit Gauthier (Editor). Sillery, Presses de l’Université du Québec. p. 329-356.

LÓPEZ HIDALGO, Antonio (2012): La columna: periodismo y literatura en un género plural. Zamora, Comunicación Social.

LAZARSFELD, Paul (1953): “The election is over”, en Public Opinion Quarterly n 53.

MARTÍN VIVALDI, Gonzalo (1987): Géneros periodisticos. Madrid, Paraninfo.

MARTÍNEZ ALBERTOS, José Luis (1983 [1992]): Curso general de Redacción periodística. Periodismo en prensa, radio, televisión y cine. Lenguaje, estilo y géneros periodísticos. Barcelona, Mitre.

MARTÍNEZ FÁBREGAS, Jezabel (2014): La imagen del Gobierno en la prensa oficial durante la transición espanyola (1975-1978). Análisis comparativo de Arriba, Pueblo, Solidaridad Nacional, El Pueblo Gallego, Hierro, Sur y Odiel. Tesis Doctoral. Sevilla, Universidad de Sevilla.

MAURI DE LOS RÍOS, Marcel (2010): Funció i evolució de la prensa de Barcelona durant la transició democrática (1975-1978). Tesis Doctoral. Barcelona, Universitat Pompeu Fabra.

MENESES, $\mathrm{M}^{\mathrm{a}}$ Dolores (2008): Noticias sobre la prensa: imagen propia en la transición democrática. Madrid, Fragua.

MONTABES PEREIRA, Juan (1986): La Prensa del Movimiento: Estudio analítico de la Prensa del Estado durante la Transición Política española 1975-1978. Tesis Doctoral. Granada, Universidad de Granada.

MONTABES PEREIRA, Juan (1989): La prensa del estado durante la transición política española. Madrid, Siglo XXI de España de Editores.

PEÑA MARÍN, Cristina (1980): El discurso politico en la prensa madrileña del franquismo. Madrid, Bulzoni.

PINEDA CACHERO, Antonio (2001): "El modelo de propaganda de Noam Chomsky: medios mainstream y control del pensamiento". En AMBITOS, n 6 , Sevilla, Universidad de Sevilla, p. 191-210.

PINEDA CACHERO, Antonio (2006). Elementos para una teoría comunicacional de la propaganda. Sevilla: Ediciones Alfar.

POSTOUTENKO, K. J. (2010). Soviet culture: codes and messages. Verlag Otto Sagner, Bielfeld.

POWELL, Charles T. (1991): El piloto del cambio: el rey, la monarquía y la transición a la democracia. Madrid, Editorial Planeta.

SEOANE, $\mathrm{M}^{\mathrm{a}}$ Cruz y SAIZ, $\mathrm{M}^{\mathrm{a}}$ Dolores (2007): Historia del Periodismo en España 3. El Siglo XX: 1896-1936. Madrid, Alianza Editorial.

SOTO, Álvaro (1998): La transición a la democracia. Madrid, Alianza Editorial. 
TUSELL, Javier (1991): La transición española a la democracia. Madrid, Colección Biblioteca Historia 16.

VILLALOBOS SALAS, Cristóbal (2010): "El articulismo de opinión como fuente para la investigación histórica: el ejemplo del franquismo". Espéculo: Revista de Estudios Literarios, $\mathrm{n}^{\circ}$ 47, p. 29. 